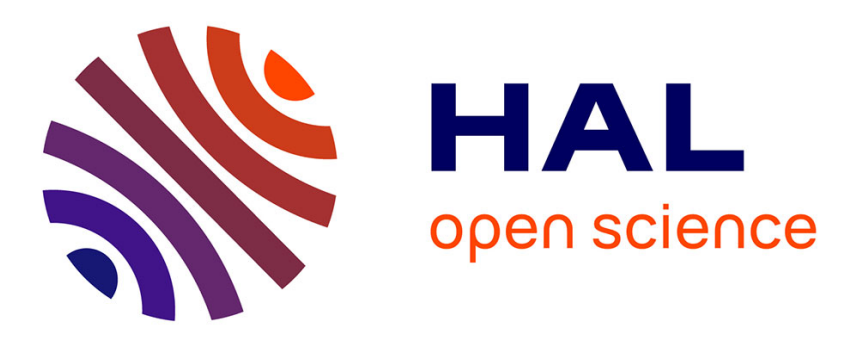

\title{
Liquid optical fibers with multistable core actuated by light radiation pressure
}

\author{
Etienne Brasselet, Regis Wunenburger, Jean-Pierre Delville
}

\section{To cite this version:}

Etienne Brasselet, Regis Wunenburger, Jean-Pierre Delville. Liquid optical fibers with multistable core actuated by light radiation pressure. Physical Review Letters, 2008, 101 (1), pp.014501/1-4. 10.1103/PhysRevLett.101.014501 . hal-00384728

\section{HAL Id: hal-00384728 \\ https://hal.science/hal-00384728}

Submitted on 15 May 2009

HAL is a multi-disciplinary open access archive for the deposit and dissemination of scientific research documents, whether they are published or not. The documents may come from teaching and research institutions in France or abroad, or from public or private research centers.
L'archive ouverte pluridisciplinaire HAL, est destinée au dépôt et à la diffusion de documents scientifiques de niveau recherche, publiés ou non, émanant des établissements d'enseignement et de recherche français ou étrangers, des laboratoires publics ou privés. 


\title{
Liquid optical fibers with multistable core actuated by light radiation pressure
}

\author{
Etienne Brasselet, Régis Wunenburger, and Jean-Pierre Delville \\ Centre de Physique Moléculaire Optique et Hertzienne, Université Bordeaux 1, \\ CNRS, 351 Cours de la Libération, 33405 Talence Cedex, France
}

(Dated: May 19, 2008)

\begin{abstract}
We report on spatio-temporal behavior of self-adapted dielectric liquid columns generated and sustained by light radiation pressure. We show that single or multi-valued liquid column diameter depends on the excitation light beam. When the beam diameter is sufficiently small, we observe a well-defined stationary column diameter. In contrast, at larger beam diameter, the liquid column experiences complex spatio-temporal dynamics whose statistical analysis evidences an underlying multistable structure. Experimental observations are all supported by a full electromagnetic model that accounts for the wave guiding properties of the liquid column viewed as a step-index liquidcore/liquid-cladding optical fiber having an optically tunable core diameter.
\end{abstract}

PACS numbers: 47.20.Ma,42.25.Gy,42.50.Wk,82.70.Kj

By combining optics and microfluidics, optofluidics becomes an emergent research field offering an unprecedented level of integration to build a new generation of microdevices merging optical reconfigurability, smoothness of fluid interfaces and the compactness of microchips 11. If great achievements were realized in optical switching [2] and splitting [3], adaptive lensing [4, 5], interferometry [ $[$ ], and lasing [7], optical guiding by liquid fibers remains very challenging because stabilization of free-standing liquid columns encounters a fundamental limitation associated to the Rayleigh-Plateau instability [8]: in weightless conditions, a liquid column breaks when its length exceeds its circumference due to capillary forces. A method involving flow focusing in microchannels has thus been developed to build liquid-core/liquidcladding waveguides [9]. It offers a good level of control since the refractive indices can be changed with the fluids, and the size and the path of the liquid core can be varied with the fluid flow rates. Tunability cannot be nevertheless instantaneous and optical guiding is not adapted to the incident light because both aspects are controlled externally by flow rates.

Strategies based on electric fields $10-12$ and acoustic radiation pressure 13. have also been adopted to bypass the Rayleigh-Plateau instability of static liquid column and have succeeded in slightly repelling the onset by less than a factor of two. A few years ago, an optical technique relying on light radiation pressure 14] has demonstrated the unique ability to stabilize liquid columns with very large aspect ratio and to offer self-adaptation to the laser wave used to form them, thus offering tunability of the diameter column and adjustment of its direction. However, no theoretical explanation has been advanced so far to describe this behavior, and the mechanism ensuring the column stability and fixing the liquid optical fiber diameter still remained open issues.

In this work, we present dedicated experiments of radiation pressure sustained liquid columns with aspect ratio up to 100 showing that either single or multi-valued col- umn diameter may exist depending on the diameter and power of the light beam. Viewing a liquid column as a step-index optical fiber having an adaptable core diameter, we build an electromagnetic model that takes into account both its optical waveguiding properties and radiation pressure effects. The model predicts (i) the stability conditions of a liquid column sustained by light, and (ii) that the mono/multi-modal character of light propagation inside the liquid fiber is associated to the existence of mono/multi-stable states. It is found to be in very good quantitative agreement with experimental observations, thus demonstrating a new mechanism of stabilization by light of two-phase liquid structures. Present results are the first demonstration of a self-adapted liquidcore/liquid-cladding optical fiber. Moreover its characteristics can be tuned by light at will.

Experiments are performed in a near-critical quaternary liquid mixture made of toluene, sodium dodecyl sulfate (SDS), n-butanol and water with weight fraction $70 \%, 4 \%, 17 \%$ and $9 \%$, respectively. Details on the preparation of the solution can be found in 15]. For a temperature above the liquid-liquid critical temperature, $T_{\mathrm{C}}=35^{\circ} \mathrm{C}$, this mixture separates in two transparent water-in-oil micellar phases labeled 1 and 2 , whose interfacial surface tension $\sigma$ vanishes as $T_{\mathrm{C}}$ is reached 15. Since density (index of refraction) of water is larger (smaller) than that of toluene, the micellar phase of larger concentration (phase 1) is located below the low micellar concentration (phase 2), while its refractive index $n_{1}$ is smaller than $n_{2}$. The experimental configuration is shown in Fig. 1(a). The mixture is enclosed in a $e=1 \mathrm{~mm}$-thick fused quartz cell that is thermally controlled at $T=T_{\mathrm{C}}+2( \pm 0.05) \mathrm{K}$ for which we have $n_{1}=1.444, n_{2}=1.460$ and $\sigma=1.75 \times 10^{-7} \mathrm{~N} / \mathrm{m}$ [16]. Close to $T_{\mathrm{C}}$ the phase 2 , of height $e / 2$, completely wets the cell walls [Fig. 1(a)].

A vertical downward linearly polarized Gaussian beam from a $\mathrm{CW} \mathrm{Ar}^{+}$laser operating at wavelength $\lambda=$ $514.5 \mathrm{~nm}$ is focused onto the interface by a microscope 


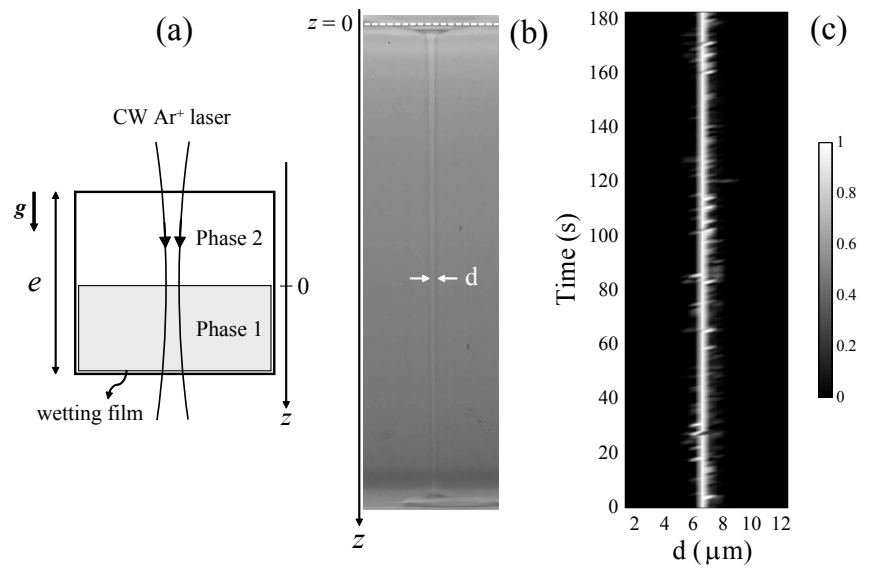

FIG. 1: (a) Experimental set-up. The TEM 00 mode of a CW Argon ion laser operating at wavelength $\lambda=514.5 \mathrm{~nm}$ is focused at the fluid-fluid interface of the phase separated liquid mixture whose temperature is regulated above the critical temperature $T_{\mathrm{c}}$. The less refractive phase, labeled 1 , is the denser one. The cell thickness is $e=1 \mathrm{~mm}$. (b) Typical liquid column with diameter $d$. (c) Dynamics of the diameter normalized probability along the column, $\Lambda_{z}(d) / \max \left(\Lambda_{z}(d)\right)$, with $w_{0}=1.8 \mu \mathrm{m}$ and $P=600 \mathrm{~mW}$.

objective ensuring a cylindrical symmetry of the intensity profile, as shown in Fig. 1(a). The intensity profile at $z=0$ (i.e. the altitude of the unperturbed interface) is $I(r)=\frac{2 P}{\pi w_{0}^{2}} \exp \left(-\frac{2 r^{2}}{w_{0}^{2}}\right)$ where $P$ is the total beam power and $w_{0}$ is the beam waist. At high enough power, typically hundreds of $\mathrm{mW}$, a perfectly beam-centered $0.5 \mathrm{~mm}$-long liquid column of phase 2 forms between the interface and the wetting layer [Fig. 1(b)] following an opto-hydrodynamic instability [17].

For the smallest beam waists we used, $w_{0}=1.8,2.7$ and $3.5 \mu \mathrm{m}$, a stable column with a well-defined diameter $d$ is observed. The diameter probability distribution along the column, $\Lambda_{z}(d)$, indeed exhibits a single valued and stationary behavior, as shown in Fig. 1(c) where $w_{0}=1.8 \mu \mathrm{m}$ and $P=600 \mathrm{~mW}$ (the acquisition rate is $25 \mathrm{~Hz}$ in all experiments). The power dependence of the column diameter for such structures having a stable and well-defined diameter is summarized in Fig. 2, where the filled and open symbols refer to increasing and decreasing power, respectively. We conclude that no memory effect is observable. This means that $d$ is unambiguously defined once $w_{0}$ and $P$ are fixed within the explored range of parameters. However, we observe a strong non-local effects since, at fixed power, the ratio $d / w_{0}$ increases when the spatial extension of the excitation beam, $w_{0}$, is decreased. Such a dependence is non-trivial and requires to look at the light-matter interaction in some details. Our approach consists of considering the column as a liquid step-index optical fiber with self-adaptable core, where phase 1 and 2 play the role of the clad and the core respectively, recalling that $n_{1}<n_{2}$. On the one hand, it is clear that such a liquid fiber does not collapse due to the radially inward acting Laplace pressure because of the radially outward competing light radiation pressure. On the other hand, it is known that the power injection and the resulting modal structure of the optical field inside a fiber strongly depends on its characteristics, namely the refractive index contrast between the clad and the core, the core diameter and the beam waist at the entrance of the fiber [18]. Therefore, in order to quantitatively describe the self-adapted liquid optical fiber sustained by light radiation pressure, one has to simultaneously solve the following coupled problems that are (i) the injection of the Gaussian incident beam into the fiber, (ii) the light propagation inside the fiber, and (iii) the balance of the involved forces. An overview of our model and its main results are given below. More details will be found in a forthcoming publication.

We consider the liquid column as a semi-infinite cylinder of radius $R=d / 2$ perfectly aligned with the incident excitation beam, the inner and outer refractive indices being $n_{2}$ and $n_{1}$, respectively. Since $n_{1} \simeq n_{2}$, the modes that propagates inside the column can be described satisfactorily by the linearly polarized $\mathrm{LP}_{l m}$ modes [18] and only the first-order terms in $n_{2}-n_{1}$ can be retained in the calculations. Since the incident beam is Gaussian, the coupling to modes with $l \geq 1$ is zero and only the $\mathrm{LP}_{0 m}$ modes are excited, each of them carrying a power $P_{m}=T_{m} P$ where $T_{m}$ is the coupling coefficient. The transmission for the $m$-th mode is calculated from the normalized overlap integral between the incident Gaussian field and the propagating $\mathrm{LP}_{0 m}$ field,

$$
T_{m}=\frac{8}{w_{0}^{2} R^{2}} \frac{\left|\int_{0}^{\infty} \mathcal{R}_{m}(r) e^{-\frac{r^{2}}{w_{0}^{2}}} r d r\right|^{2}}{\frac{J_{1}^{2}\left(\kappa_{m} R\right)}{J_{0}^{2}\left(\kappa_{m} R\right)}+\frac{K_{1}^{2}\left(\gamma_{m} R\right)}{K_{0}^{2}\left(\gamma_{m} R\right)}},
$$

where $J_{n}$ and $K_{n}$ are the usual Bessel functions, $\kappa_{m}$ and $\gamma_{m}$ are the $m$-th roots of the characteristic equation that defines the $\mathrm{LP}_{0 m}$ mode, $\kappa_{m} \frac{J_{1}\left(\kappa_{m} R\right)}{J_{0}\left(\kappa_{m} R\right)}=\gamma_{m} \frac{K_{1}\left(\gamma_{m} R\right)}{K_{0}\left(\gamma_{m} R\right)}$ where $\left(\kappa_{m} R\right)^{2}+\left(\gamma_{m} R\right)^{2}=(2 \pi / \lambda)^{2}\left(n_{2}^{2}-n_{1}^{2}\right)$ [18]. The function $\mathcal{R}_{m}(r)$ is associated to the radial profile of the electric field of the mode $m$,

$$
\mathcal{R}_{m}(r)=\left(\left.\frac{J_{0}\left(\kappa_{m} r\right)}{J_{0}\left(\kappa_{m} R\right)}\right|_{r \leq R},\left.\frac{K_{0}\left(\gamma_{m} r\right)}{K_{0}\left(\gamma_{m} R\right)}\right|_{r \geq R}\right)
$$

In weightless conditions for steady and perfectly cylindrical columns, the interface equilibrium condition writes $\Pi_{\text {radiation }}=\Pi_{\text {Laplace }}$, where $\Pi_{\text {Laplace }}=\sigma / R$, since the viscous stress due to a possible flow within a perfect cylinder is tangential to the interface. The radiation pressure $\Pi_{\text {radiation }}$ corresponds to the radial discontinuity of the electromagnetic stress tensor 19] across the normal to the interface. In the present situation involving non mag- 


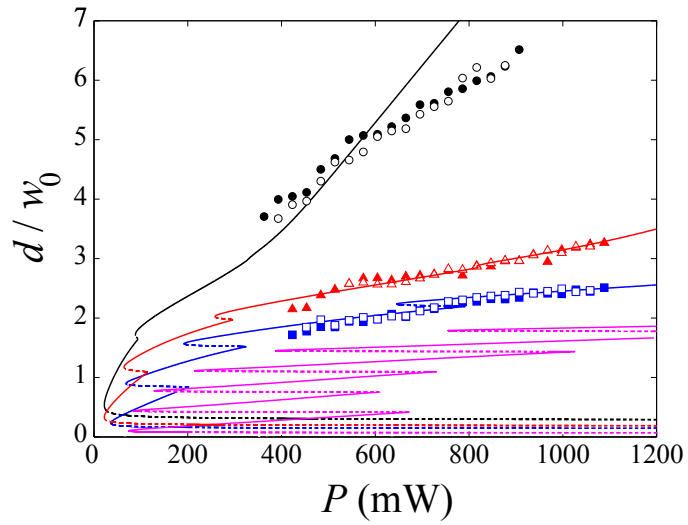

FIG. 2: (Color online) Power dependence of the liquid fiber diameter for beam waists $w_{0}=1.8 \mu \mathrm{m}$ (black line and circles), $2.7 \mu \mathrm{m}$ (red line and triangles), $3.5 \mu \mathrm{m}$ (blue line and squares) and $7.0 \mu \mathrm{m}$ (magenta). Filled and open symbols refer to increasing and decreasing power, respectively. Solid and dashed lines corresponds respectively to stable and unstable states predicted by the model.

netic fluids, to the lowest order in $n_{2}-n_{1}$, we get

$$
\Pi_{\text {radiation }}=\frac{1}{2} \epsilon_{0} \bar{n}\left(n_{2}-n_{1}\right) \sum_{m}\left|\mathbf{E}^{(m)}\right|_{r=R}^{2},
$$

where $\bar{n}=\left(n_{2}+n_{1}\right) / 2$ is the averaged refractive index, $\epsilon_{0}$ the vacuum permittivity and $\mathbf{E}^{(m)}$ the complex electric field of the mode $m$. Then, since the flux of the $z$-component of the Poynting vector across a plane perpendicular to the $z$-axis equals the power carried by the $m$-th mode, $P_{m}=T_{m} P$, we deduce

$$
\left|\mathbf{E}^{(m)}\right|_{r=R}^{2}=\frac{2 P_{m}}{\epsilon_{0} \pi c \bar{n} R^{2}}\left(\frac{J_{1}^{2}\left(\kappa_{m} R\right)}{J_{0}^{2}\left(\kappa_{m} R\right)}+\frac{K_{1}^{2}\left(\gamma_{m} R\right)}{K_{0}^{2}\left(\gamma_{m} R\right)}\right)^{-1}
$$

Finally $\Pi_{\text {radiation }}$ is explicitly obtained by combining Eqs. (1), (3) and (4).

The column diameter is further found by numerically solving the equilibrium equation. The model predicts that there is no equilibrium radius below a critical power and a discrete set of solutions $\left\{R_{\mathrm{eq}}^{(n)}\right\}$ above. The stability criterion is obtained from standard statics stability analysis at $R=R_{\text {eq }}^{(n)}$ and leads to $\partial \Pi_{\text {radiation }} / \partial R<$ $\partial \Pi_{\text {Laplace }} / \partial R$. Results are displayed in Fig. 2 where solid (dashed) line refers to stable (unstable) solutions. As far as the smallest waists are considered (i.e. like those presented in Fig. 2) a good quantitative agreement with experimental data is obtained, especially since no adjustable parameter is used. A more complex sequence of stable and unstable states is predicted at lower power but this region cannot be explored experimentally since the large aspect ratio column detaches from the wetting film at the bottom of the cell. Note finally that a single and well-defined diameter does not imply a monomodal (i.e. $m=1$ only) behavior. Indeed, as an example, the numbers of modes involved in the range of parameters presented in Fig. 2 is $m_{\max }=6$ and 4 for $w_{0}=1.8$ and $2.7 \mu \mathrm{m}$, respectively.

When the beam waist is sufficiently large, the model predicts multistable states as shown in Fig. 2 where the results for $w_{0}=7 \mu \mathrm{m}$ are shown in magenta. There are indeed several stable radii at fixed power that should correspond to several observable column diameters. One would expect to experimentally observe metastable states as well as a hysteretic behavior of $d(P)$. We rather observe a column whose diameter varies along $z$ and with time $t$, in distinction to what is found for smaller waist [Fig. 1(b,c)]. This point is detailed in Fig. 3 for $w_{0}=7 \mu \mathrm{m}, P=575 \mathrm{~mW}$ at a fixed time. The column diameter as a function of $z$ is shown in panel (a), the corresponding picture being shown in panel (b). The instantaneous diameter probability distribution $\Lambda_{z}(d)$ along the column is shown in panel (c). The distribution $\Lambda_{z}(d)$ is obtained using the fit of $d(z)$ shown in panel (a), which appears as a red solid line. Typically, $\Lambda_{z}(d)$ exhibits two distinct peaks, labeled $d_{1}$ and $d_{2}$ in Fig. 3(c). Note that $d_{1,2} \sim 1.5-2 w_{0}$ and their difference $\left(d_{2}-d_{1}\right) \sim 0.4 w_{0}$ compare well to the predicted values shown in Fig. 2. The system explores different discrete diameter values at a single time, which is the signature of the bistability (or multistability) predicted by our steady state model. The mean amplitude of thermal fluctuations of the local interface position, $\left(k_{\mathrm{B}} T / \sigma\right)^{1 / 2} \sim 0.1-0.2 \mu \mathrm{m}[20]$ where $k_{\mathrm{B}} T$ is the thermal energy, is one order of magnitude smaller that the inter-plateaux distance, thus eliminating departure from a perfect cylinder by pure thermal activation. However, the coupling between these thermal fluctuations and the weak light-induced flow inside the liquid column 21] can give birth to a viscous stress normal to the interface and the subsequent exploration of multivalued fiber radii for large beam diameters. This
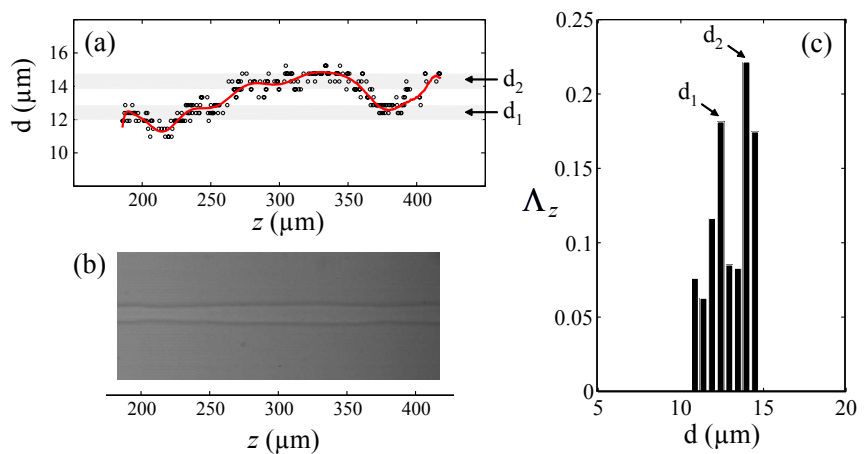

FIG. 3: (Color online) (a) Column diameter along the $z$-axis. Symbols refer to image analysis contour detection and the red line is a suitable smooth fit of experimental data. (b) Picture that corresponds to (a). (c) Diameter probability distribution along the column, at a fixed time, using the fitted diameter data shown in panel (a). The beam waist is $w_{0}=7.0 \mu \mathrm{m}$ and $P=575 \mathrm{~mW}$. 

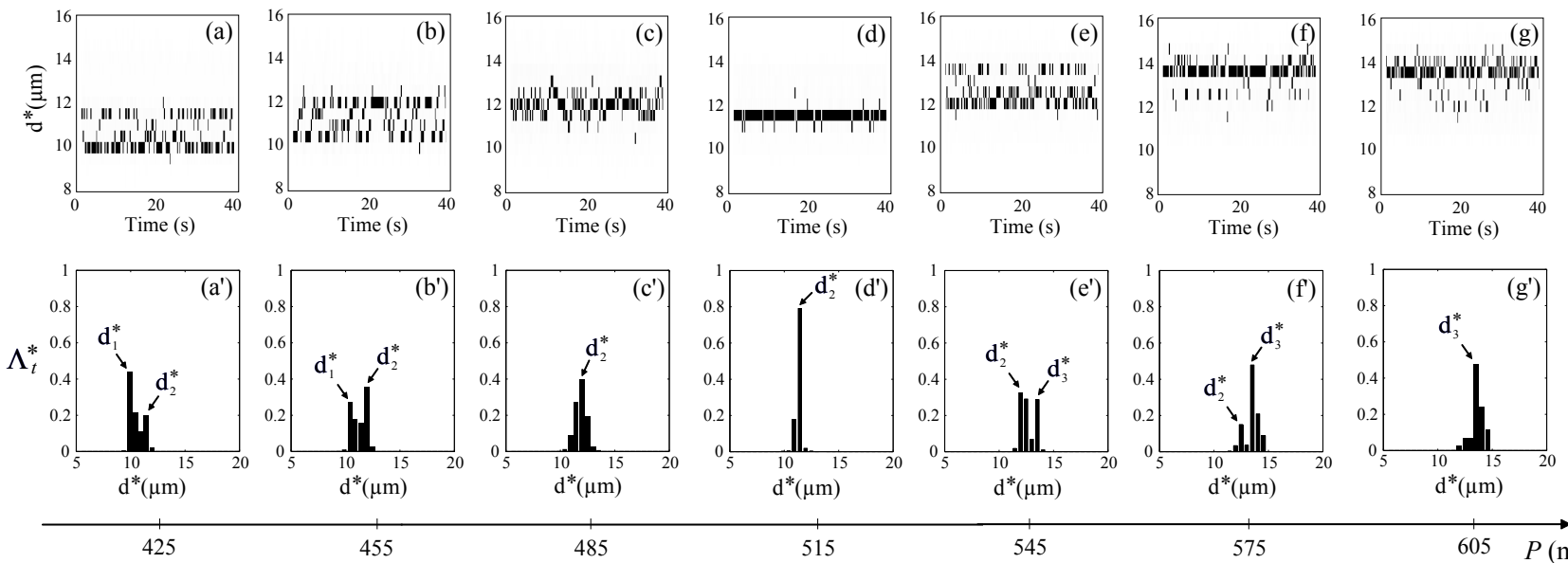

455

485

515

545

575

$605 P(\mathrm{~mW})$

FIG. 4: Upper panels: dynamics of the most probable diameter $d^{*}$ with $w_{0}=7.0 \mu \mathrm{m}$. Bottom panels: Probability distribution of $d^{*}$ over a time window of $200 \mathrm{~s}$. Panels (a,a') to (g,g') correspond to powers $P=425,455,485,515,545,575$ and $615 \mathrm{~mW}$, respectively.

signature is further strengthened by the overall increase of the set of discrete diameter values with power, which we study below by taking advantage of the long-term stationarity of the system to improve the signal-to-noise ratio of the diameter statistical distribution.

To this purpose we introduce the most probable diameter $d^{*}$ as the diameter corresponding to the maximum of $\Lambda_{z}(d)$ at a fixed time $t$. For example, we have $d^{*}=d_{2}$ for the picture analyzed in Fig. 3(c). By doing so, from a movie of the time-dependent column recorded at fixed power, we construct a time dependent signal of the most probable column diameter, $d^{*}(t)$. The power dependence of $d^{*}(t)$ is shown in panels (a-g) of Fig. 4. As said above, we observe that the trend of $d^{*}(t)$ is to increase with $P$ in agreement with the statics model tendency.

Interestingly, depending on the power, $d^{*}(t)$ either is almost time independent [panels $(\mathrm{d}, \mathrm{g})$ of Fig. 4] or exhibits a double-valued behavior [panels (a, b, c, e and f) of Fig. 4]. This is supported by the aspect and the power dependence of the probability distribution of $d^{*}(t)$, $\Lambda_{t}^{*}\left(d^{*}\right)$, shown in panels (a'-g') of Fig. 4. As $P$ is increased from 425 [Fig. 4(a)] to $515 \mathrm{~mW}$ [Fig. 4(d)], the height of the probability peak corresponding to $d_{1}^{*}$ progressively decreases while the one corresponding to $d_{2}^{*}$ increases. The same behavior is observed between 515 and $605 \mathrm{~mW}$, showing the reproducibility of this trend. So, in experimental conditions for which the steady-state equilibrium model predicts a multistable behavior of $d$ versus $P$ resulting from successive subcritical bifurcations, the observed most probable column diameter $d^{*}$ is time-dependent and takes only discrete values. Only the probability of observation of these discrete $d^{*}$ values continuously depends on $P$. This statistical analysis allowed by the time dependence of the columns diameter thus evidences the underlying multistable behavior predicted by the steady-state equilibrium model. It also explains why no diameter hysteresis could be observed. In fact the observed interface fluctuations make the column investigate its allowed steady states, thus preventing the column from staying in a metastable state, in a very similar way to noise-induced bistability in presence of a subcritical bifurcation [22].

In conclusion, we have demonstrated the mechanism by which liquid columns of large aspect ratio can be sustained by light. We showed that column stability occurs above a beam power threshold and described the self-adaptation to the incident exciting light wave. At large beam waist this adaptation becomes multivalued in agreement with experiments. The column stabilization well above the onset of the Rayleigh-Plateau instability and the self-adaptation of the liquid fiber core diameter to the incident beam whatever its power and waist open a promising route toward reconfigurable light guiding applications of great versatility.

[1] D. Psaltis, S. R. Quake and C. Yang, Nature 442, 381 (2006).

[2] K. Campbell et al., Appl. Phys. Lett. 85, 6119 (2004).

[3] N. T. Nguyen et al., J. Micromech. Microeng. 17, 2169 (2007).

[4] A. Casner, J. P. Delville, Optics Lett. 26, 1418 (2001).

[5] D. Y. Zhang, N. Justis, and Y. H. Lo, Appl. Phys. Lett. 84, 4194 (2004).

[6] C. Grillet et al., Opt. Express 12, 5440 (2004).

[7] Z. Li et al., Opt. Express 14, 696 (2006).

[8] J. Eggers, Rev. Mod. Phys. 69, 865 (1997).

[9] D. B. Wolfe et al., Proc. Natl Acad. Sci. USA 101, 12434 (2004).

[10] H. Gonzalez et al., J. Fluid Mech. 206, 545 (1989).

[11] C. L. Burcham and D. A. Saville, J. Fluid Mech. 40537 (2000). 
[12] M. J. Marr-Lyon et al., Phys. Fluids 12986 (2000).

[13] M. J. Marr-Lyon, D. B. Thiessen and P. L. Marston, Phys. Rev. Lett. 86, 2293 (2001).

[14] A. Casner and J. P. Delville, Europhys. Lett. 65, 337 (2004).

[15] A. Casner and J. P. Delville, Phys. Rev. Lett. 87, 054503 (2001).

[16] H. Chrabi et al., European Journal of Mechanics B/Fluids, in press (2008).

[17] A. Casner and J. P. Delville, Phys. Rev. Lett. 90, 144503
(2003).

[18] K. Okamoto, Fundamentals of Optical Waveguides, 2nd ed. Elsevier, Amsterdam, 2006.

[19] L. Landau and E. Lifschitz, Electrodynamics of Continuous Media, Pergamon Press, Oxford, 1984.

[20] D. G. A. L. Aarts, M. Schmidt, and H. N. W. Lekkerkerker, Science 304, 847 (2004).

[21] R. D. Schroll et al., Phys. Rev. Lett. 98, 133601 (2007).

[22] S. Residori et al., Phys. Rev. Lett. 88, 024502 (2001). 